\title{
BMJ Open Risk factors for drug-resistant tuberculosis, the association between comorbidity status and drug-resistant patterns: a retrospective study of previously treated pulmonary tuberculosis in Shandong, China, during 2004-2019
}

\author{
Ning-ning Tao (D) , ${ }^{1,2}$ Yi-fan $\mathrm{Li}^{1,}{ }^{1,2}$ Wan-mei Song (D) , ${ }^{1}$ Jin-yue Liu, ${ }^{3}$ \\ Qian-yun Zhang, ${ }^{1}$ Ting-ting Xu, ${ }^{2}$ Shi-jin Li, ${ }^{1}$ Qi-qi An, ${ }^{1}$ Si-qi Liu, ${ }^{1}$ Huai-chen Li ${ }^{1,2,4}$
}

To cite: Tao N, Li Y, Song W, et al. Risk factors for drugresistant tuberculosis, the association between comorbidity status and drug-resistant patterns: a retrospective study of previously treated pulmonary tuberculosis in Shandong, China, during 2004-2019. BMJ Open 2021;11:e044349. doi:10.1136/ bmjopen-2020-044349

- Prepublication history for this paper is available online. To view these files, please visit the journal online (http://dx.doi. org/10.1136/bmjopen-2020044349).

$\mathrm{N}-\mathrm{nT}$ and $\mathrm{Y}$-fL contributed equally.

Received 07 September 2020 Accepted 18 May 2021

Check for updates

(c) Author(s) (or their employer(s)) 2021. Re-use permitted under CC BY-NC. No commercial re-use. See rights and permissions. Published by BMJ.

For numbered affiliations see end of article.

Correspondence to Professor Huai-chen Li; lihuaichen@163.com

\section{ABSTRACT}

Objective This study was designed to identify the risk factors for drug-resistant tuberculosis (DR-TB) and the association between comorbidity and drug resistance among retreated pulmonary tuberculosis (PTB).

Design A retrospective study was conducted among all the 36 monitoring sites in Shandong, China, over a 16-year period. Baseline characteristics were collected from the TB Surveillance System. Categorical variables were compared by Fisher's exact or Pearson's $\chi^{2}$ test. The risk factors for drug resistance were identified using univariable analysis and multivariable logistic models. The influence of comorbidity on different types of drug resistance was evaluated by performing multivariable logistic models with the covariates adjusted by age, sex, body mass index, drinking/smoking history and cavity.

Results A total of 10975 patients with PTB were recorded during 2004-2019, and of these 1924 retreated PTB were finally included. Among retreated PTB, 26.2\% were DR-TB and $12.5 \%$ had comorbidity. Smoking (adjusted OR (aOR): $1.69,95 \% \mathrm{Cl} 1.19$ to 2.39 ), cavity (aOR: $1.55,95 \% \mathrm{Cl} 1.22$ to 1.97 ) and comorbidity (aOR: $1.44,95 \% \mathrm{Cl} 1.02$ to 2.02 ) were risk factors for DR-TB. Of 504 DR-TB, 9.5\% had diabetes mellitus, followed by hypertension $(2.0 \%)$ and chronic obstructive pulmonary disease (1.8\%). Patients with retreated PTB with comorbidity were more likely to be older, have more bad habits (smoking, alcohol abuse) and have clinical symptoms (expectoration, haemoptysis, weight loss). Comorbidity was significantly associated with DR-TB (aOR: $1.44,95 \% \mathrm{Cl} 1.02$ to 2.02 ), overall rifampin resistance (aOR: $2.17,95 \% \mathrm{Cl} 1.41$ to 3.36 ), overall streptomycin resistance (aOR: $1.51,95 \% \mathrm{Cl} 1.00$ to 2.27$)$ and multidrug resistance (aOR: 1.96, 95\% $\mathrm{Cl} 1.17$ to 3.27 ) compared with pan-susceptible patients $(p<0.05)$.

Conclusion Smoking, cavity and comorbidity lead to an increased risk of drug resistance among retreated PTB. Strategies to improve the host's health, including smoking cessation, screening and treatment of comorbidity, might
Strengths and limitations of this study

- This study had a large sample size and long time span.

- The sample on the association between comorbidity status and drug-resistant tuberculosis among patients with retreated pulmonary tuberculosis in Shandong Province, China, is representative.

- The diversity in diagnostic and therapeutic levels from different tuberculosis monitoring sites may lead to bias.

- The diagnosis of tuberculosis based on microscopy inevitably underestimated the burden of tuberculosis.

contribute to the control of tuberculosis, especially DR-TB, in China.

\section{INTRODUCTION}

With the changing of demographics and lifestyle, the spectrum of disease has been transformed from infectious diseases to noncommunicable diseases (NCDs). ${ }^{1}$ However, people from developing countries suffer the double burden of infectious diseases and NCDs. ${ }^{2}$ As an infectious disease, tuberculosis (TB) can be prevented and treated well. Although the control of TB has achieved considerable progress in the past decades, this seems to have reached its bottleneck recently with 1.45 million deaths due to TB, which ranks the topmost cause of death among infectious agents. ${ }^{3}$ The overlapping $\mathrm{TB}$ and comorbidities exacerbate the risk and mortality of the other. ${ }^{1}$ The bidirectional 
deleterious correlation between $\mathrm{TB}$ and coexisting diseases might open a new direction for future TB control.

Drug resistance is an intractable public problem and a crucial obstacle to TB control. According to a 2019 global TB report, about half a million new cases were rifampicinresistant TB (RR-TB), among which $78 \%$ cases were multidrug-resistant TB (MDR-TB), a kind of resistance to both isoniazid (INH) and rifampicin (RFP). Drug resistance not only was an indicator of poor outcomes, but it also resulted in fewer effective drugs to choose, higher expenses to pay, and the spread and exacerbation of drug-resistant TB (DR-TB). ${ }^{45}$ Patients with previous anti-TB treatment are at high risk of developing DR-TB. Compared with newly treated TB cases $(3.4 \%)$, the rate of MDR-TB/RR-TB was $18 \%$ among retreated cases. ${ }^{3}$ The control of DR-TB especially those among retreated patients is imperative.

Various studies and reviews reported that host factors including smoking, alcohol abuse, low body mass index (BMI), comorbidity (eg, HIV infection, diabetes mellitus (DM), chronic renal failure (CRF), malignancy, chronic obstructive pulmonary disease (COPD), silicosis) can predispose to the development of TB.$^{6-12}$ Several of these factors were associated with poor treatment outcomes (eg, alcohol abuse, HIV infection, DM) ${ }^{13}{ }^{14}$ TB relapse (eg, HIV infection, DM $)^{13} 15$ and the development of MDR-TB (eg, alcohol abuse, HIV infection, DM, COPD) significantly. ${ }^{616} 17$ Coexisting diseases are continuously being identified as a vital factor in the control of TB. It is believed that the improvement of the host's health status, both timely identification and effective treatment of comorbidity, may alleviate the development of TB and reduce the spread of DR-TB.

Although China is an upper-middle-income country, with half its population residing in urban areas, the burden of DR-TB (only followed behind by India) and NCDs is very serious. This study aims to summarise the characteristics of host status and types of drug resistance in retreated pulmonary $\mathrm{TB}$ (PTB), identify the risk factors for drug resistance of these patients, and evaluate the contribution of comorbidity to different types of drug resistance among retreated PTB in Shandong Province, China, during 2004-2019.

\section{METHODS \\ Setting}

This retrospective cohort study was conducted in the second most populous province of China, Shandong Province. In 2019, about 100.47 million populations resided in an area of $157100 \mathrm{~km}^{2}$ in Shandong Province, which is located at $36^{\circ} 24^{\prime} \mathrm{N}$ latitude, $118^{\circ} 24^{\prime} \mathrm{E}$ longitude, with 17 municipalities and 137 counties (districts) (http://www.stats-sd.gov.cn/).

\section{Study population and data collection}

In Shandong Province, there are 13 municipal-level local health departments, 2 province-level and 21 county-level hospitals, which were responsible for quality assessment in

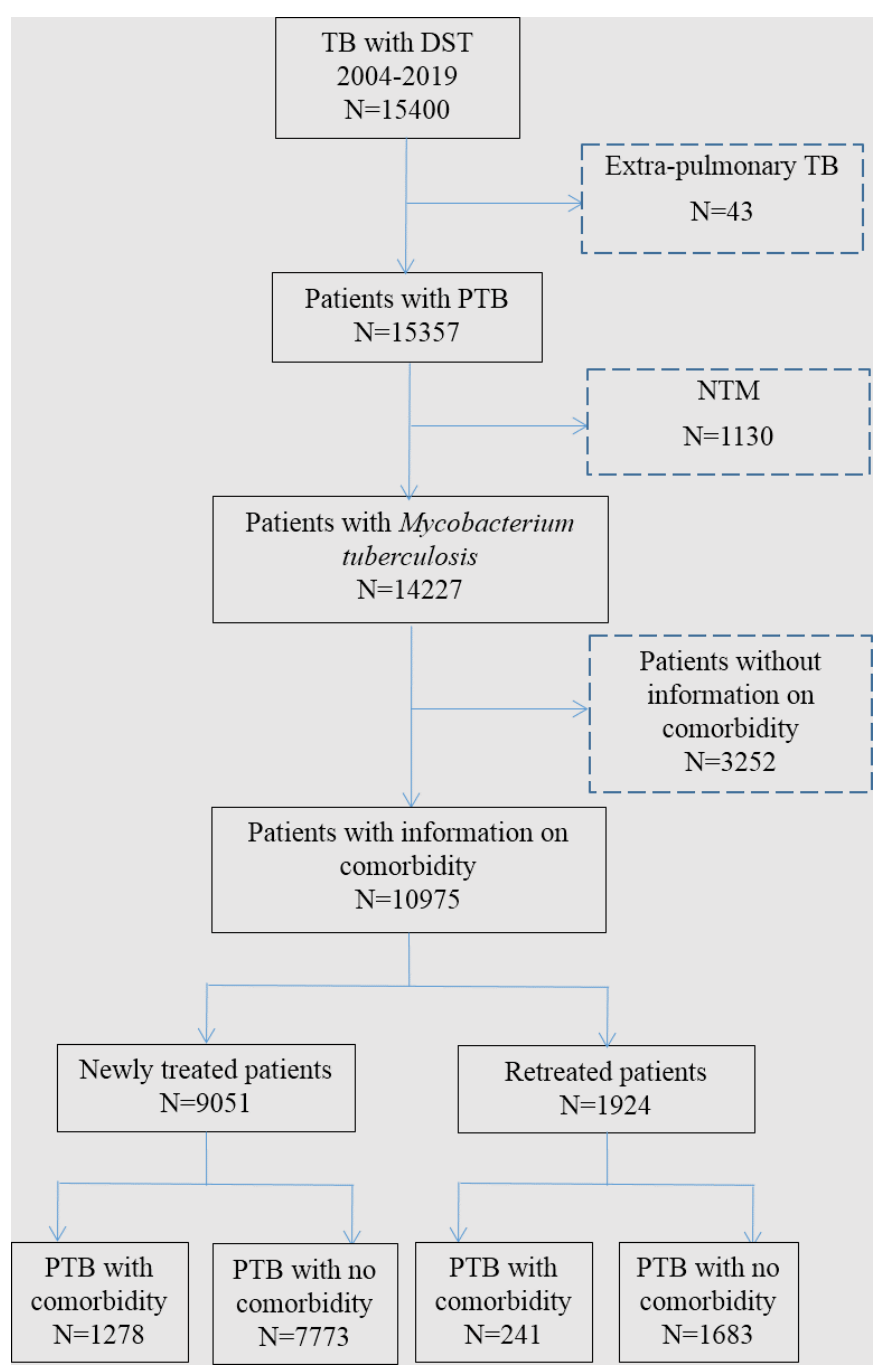

Figure 1 TB cases in Shandong, China. DST, drug susceptibility testing; NTM, non-tuberculous mycobacteria; PTB, pulmonary tuberculosis TB, tuberculosis.

TB surveillance. We searched the TB Surveillance System in Shandong and collected information on patients with PTB with full data on comorbidity status and drug susceptibility testing (DST) results (at least for all of the four first-line anti-TB drugs) during 2004-2019. We ruled out those patients without information on comorbidity status and those with extrapulmonary TB or non-tuberculosis mycobacteria infection (figure 1). A total of 9051 newly treated and 1924 retreated PTB cases were identified. Of all the patients with retreated PTB with Mycobacterium tuberculosis infection, 1683 had no comorbidity and 241 had at least one comorbidity. Demographic information (age and sex) and clinical information (BMI, smoking, alcohol abuse, cavity and symptoms) were collected.

\section{Laboratory diagnosis and DST}

All samples available from suspicious patients were collected by the specialist at each surveillance site. One patient should have at least two sputum samples for examinations of bacteriological culture, species identification and DST. Smear microscopy with Ziehl-Neelsen staining was performed to 
identify acid-fast bacilli. Each sample was inoculated into tubes with acidified Löwenstein-Jensen medium for further culture. ${ }^{18}$ Subsequently, the samples with growing colonies were tested for strain identification and DST. The identification of M. tuberculosis included a comprehensive consideration of the results according to p-nitrobenzoic acid, 2-thiophene carboxylic acid hydrazide testing and 16S rRNA gene sequence analysis. ${ }^{19}$

DST for first-line anti-TB drugs was performed using the proportion method on Löwenstein-Jensen medium with the following drug concentrations: INH $(0.2 \mu \mathrm{g} /$ $\mathrm{mL})$, RFP $(40 \mu \mathrm{g} / \mathrm{mL})$, ethambutol (EMB, $2.0 \mu \mathrm{g} /$ $\mathrm{mL}$ ) and streptomycin (SM, $4.0 \mu \mathrm{g} / \mathrm{mL}$ ). DST for other anti-TB drugs, such as pyrazinamide, fluoroquinolone and kanamycin, was performed according to patients' choice, which was non-routinely.

\section{Quality control}

All procedures during TB surveillance were carried out according to the WHO guidelines. External quality assessment for all laboratory tests, including smear, culture and DST, was supervised by the Superior TB National Reference Laboratory in Katharine Hsu Center of Shandong Province. ${ }^{18}$ Quality assessment and data extraction were accomplished by at least two researchers who were trained professionally.

\section{Definitions}

Drug-susceptible TB was defined as being susceptible to all of the four first-line anti-TB drugs. DR-TB were classified into monoresistance (MR), resistant to only one first-line anti-TB drug; multidrug resistance (MDR), resistant to at least both INH and RFP; and polydrug resistance (PDR), resistant to at least two first-line anti-TB drug, except to both INH and RFP. Retreated TB referred to patients who had accepted 1 month of anti-TB drugs before.

Comorbidity data collected in this study were DM, hypertension, hepatitis, CRF, connective tissue disease (CTD), disability, malignancy, HIV infection, silicosis, asthma, COPD and bronchiectasia co-occurring with TB. Comorbidity status was confirmed mainly in two ways: (1) self-reported by the patient with a previous diagnosis certificate; and (2) newly identified cases according to associated diagnostic consensus unified globally.

\section{Statistical analysis}

Continuous variables such as age were summarised with mean and SD; categorical variables including sex, BMI $(<18.5,18.5-24.9, \geq 25)$, drinking history, smoking history, TB contact history, cavity, symptoms (cough, expectoration, fever, night sweating, fatigue, haemoptysis, weight loss and chest pain) and comorbidities (silicosis, asthma, COPD, bronchiectasia, lung cancer, DM, hypertension, gastrointestinal cancer, hepatitis, renal failure, CTD and other malignancy) were summarised as proportions. Univariable analysis and multivariable logistic models were applied to identify the risk factors for drug resistance among newly treated or retreated PTB cases. Demographic characteristics, clinical traits and types of drug resistance were compared according to comorbidity status using Fisher's exact or Pearson's $\chi^{2}$ test. Multivariable logistic models were also used to estimate the influence of comorbidity on different types of drug resistance with the covariates adjusted by age, sex, BMI, drinking history, smoking history and cavity according to published research. A two-sided $\mathrm{p}<0.05$ was considered significant. All statistical analyses were calculated using SPSS V.20.0 software.

\section{RESULTS}

\section{Case estimates and risk factors for DR-TB}

The baseline characteristics of the study populations are demonstrated in table 1. A total of 10975 patients with PTB aged $49.8 \pm 19.7$ were reported in Shandong, China, in 2004-2019, of whom $9051(82.5 \%)$ cases were newly treated and 1924 (17.5\%) were retreated PTB. Among these retreated PTB cases, 26.2\% were drugresistant, $82.7 \%$ were male, $18.5 \%$ were drinkers, $25.2 \%$ were smokers, $46.4 \%$ with baseline cavity and $16.3 \%$ had comorbidity.

In all retreated PTB cases, the following characteristics were associated with the presence of DR-TB: (1) smoking (adjusted OR (aOR): 1.69, 95\% CI 1.19 to 2.39 ); (2) cavity (aOR: $1.55,95 \%$ CI 1.22 to 1.97 ); and (3) comorbidity (aOR: $1.44,95 \%$ CI 1.02 to 2.02). In all newly treated PTB cases, male sex (aOR: $1.25,95 \%$ CI 1.03 to 1.51 ) and cavity (aOR: $1.15,95 \%$ CI 1.01 to 1.31 ) were associated with the presence of DR-TB.

\section{Demographic and clinical characteristics of retreated PTB}

A total of $241(12.5 \%)$ patients with retreated PTB with comorbidity (group A) and 1683 (87.5\%) with no comorbidity (group B) were enrolled in this study. According to Pearson's $\chi^{2}$ test, patients with retreated PTB with comorbidity were more likely than those without comorbidity to be older (A vs B: $60.1 \pm 15.9$ vs $49.1 \pm 19.6$, $\mathrm{p}<0.001$ ), to be male ( $\mathrm{A}$ vs $\mathrm{B}: 87.1 \%$ vs $80.9 \%$, $\mathrm{p}<0.001$ ), with $\mathrm{BMI} \geq 25$ (A vs B: $7.9 \%$ vs $3.4 \%, \mathrm{p}=0.02$ ), to abuse alcohol (A vs $\mathrm{B}$ : $24.9 \%$ vs $17.0 \%, \mathrm{p}=0.003$ ), to be a smoker ( $\mathrm{A}$ vs $\mathrm{B}: 30.0 \%$ vs $19.5 \%, \mathrm{p}<0.001$ ), to have cavity (A vs B: $52.5 \%$ vs $35.1 \%$, $\mathrm{p}<0.001$, and to have more symptoms including expectoration (A vs B: $85.1 \%$ vs $77.2 \%, \mathrm{p}=0.006$ ), haemoptysis (A vs $\mathrm{B}: 22.0 \%$ vs $12.7 \%, \mathrm{p}<0.001$ ) and weight loss (A vs $\mathrm{B}$ : $19.5 \%$ vs $13.5 \%, \mathrm{p}=0.012$ ) (table 2 ).

\section{Drug-resistant profiles of retreated PTB}

About $34.0 \%$ (82) of patients with retreated PTB with comorbidity and $25.1 \%$ (422) without comorbidity had DR-TB $(\mathrm{p}=0.003)$. After further dividing into different drug-resistant subgroups, it showed that the rates of overall INH resistance ( $A$ vs $B: 22.8 \%$ vs $16.0 \%, p=0.008$ ), overall RFP resistance (A vs B: $19.5 \%$ vs $10.8 \%, \mathrm{p}<0.001$ ), MR to INH (A vs B: $3.7 \%$ vs $1.4 \%, p=0.007$ ), PDR to RFP+SM (A vs $B: 1.2 \%$ vs $0.2 \%, p=0.029$ ), MDR ( $A$ vs $B: 12.9 \%$ vs $7.7 \%$, $\mathrm{p}=0.006$ ) and resistance to $\mathrm{INH}+\mathrm{RFP}+\mathrm{SM}$ (A vs $\mathrm{B}: 6.6 \%$ vs $3.6 \%, \mathrm{p}=0.026$ ) were much higher in group $\mathrm{A}$ than in 


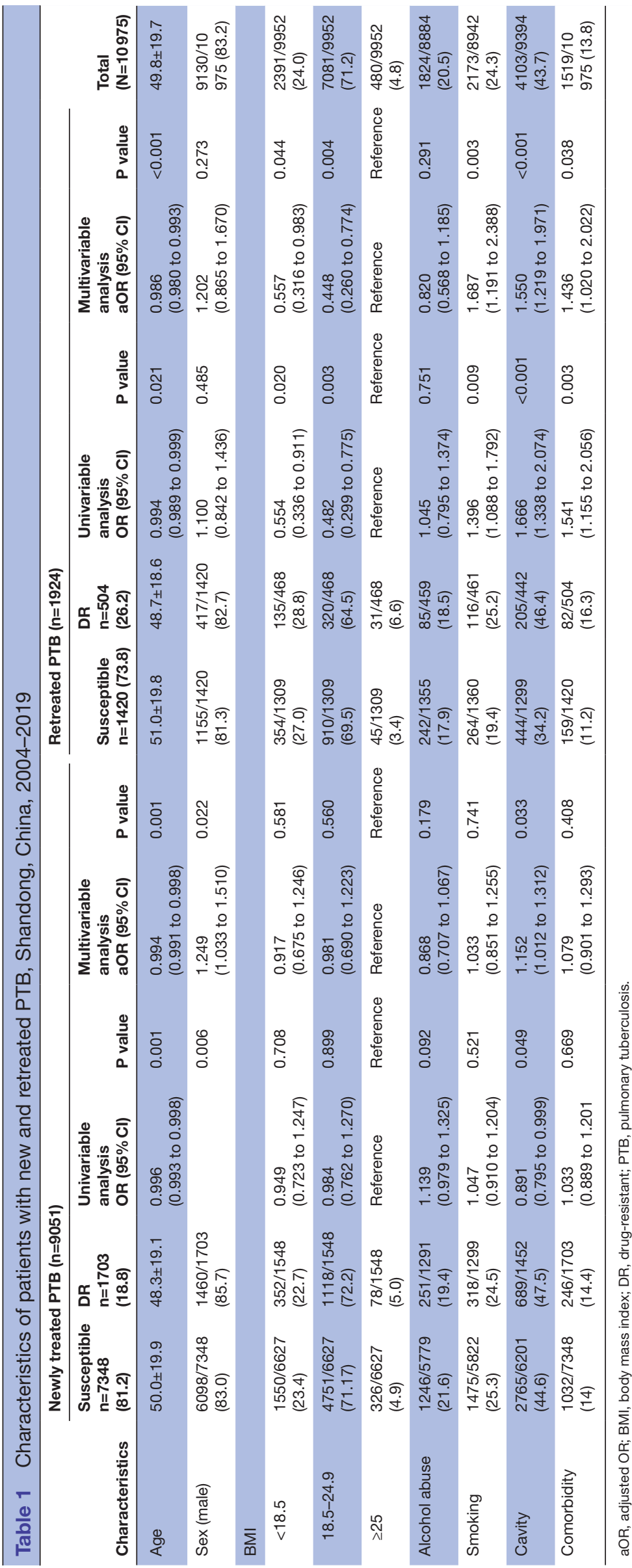


Table 2 Demographic and clinical characteristics of 1924 patients with retreated PTB

\begin{tabular}{|c|c|c|c|c|}
\hline Characteristics & $\begin{array}{l}\text { Total } \\
n=1924\end{array}$ & $\begin{array}{l}\text { Patients with PTB with } \\
\text { comorbidity } \\
\mathrm{n}=241\end{array}$ & $\begin{array}{l}\text { Patients with PTB } \\
\text { without comorbidity } \\
n=1683\end{array}$ & $P$ value \\
\hline Age & $50.43 \pm 19.53$ & $60.05 \pm 15.87$ & $49.05 \pm 19.62$ & $<0.001$ \\
\hline $0-14$ & $4(0.2)$ & $0(0)$ & $4(0.2)$ & 1 \\
\hline $15-44$ & 737 (38.3) & $42(17.4)$ & 695 (41.3) & $<0.001$ \\
\hline $45-64$ & $661(34.4)$ & $99(41.1)$ & $562(33.4)$ & 0.019 \\
\hline$>65$ & $522(27.1)$ & $100(41.5)$ & $422(25.1)$ & $<0.001$ \\
\hline Sex (male) & $1572(81.7)$ & $210(87.1)$ & 1362 (80.9) & 0.02 \\
\hline \multicolumn{5}{|l|}{ BMI (n=1777/230/1547) } \\
\hline$<18.5$ & $489(25.4)$ & $56(23.2)$ & $433(25.7)$ & Reference \\
\hline $18.5-24.9$ & $1212(63.0)$ & $155(64.3)$ & $1057(62.8)$ & 0.449 \\
\hline$\geq 25$ & $76(4.0)$ & $19(7.9)$ & $57(3.4)$ & $<0.001$ \\
\hline $\begin{array}{l}\text { Alcohol abuse } \\
(n=1814 / 233 / 1581)\end{array}$ & $327(18.0)$ & $58(24.9)$ & $269(17.0)$ & 0.003 \\
\hline Smoking ( $n=1821 / 233 / 1588)$ & 380 (20.9) & $70(30.0)$ & 310 (19.5) & $<0.001$ \\
\hline \multicolumn{5}{|l|}{ Symptom } \\
\hline Cough & $1840(95.6)$ & $226(93.8)$ & $1614(95.9)$ & 0.131 \\
\hline Expectoration & 1504 (78.3) & $205(85.1)$ & 1299 (77.2) & 0.006 \\
\hline Fever & 901 (46.8) & $104(43.2)$ & $797(47.4)$ & 0.221 \\
\hline Night sweat & $434(22.6)$ & $45(18.7)$ & $389(23.1)$ & 0.123 \\
\hline Fatigue & 756 (39.3) & 90 (37.3) & 666 (39.6) & 0.508 \\
\hline Haemoptysis & $266(13.8)$ & $53(22.0)$ & $213(12.7)$ & $<0.001$ \\
\hline Weight loss & $274(14.2)$ & 47 (19.5) & 227 (13.5) & 0.012 \\
\hline Chest pain & $217(11.3)$ & $32(13.3)$ & $185(11.0)$ & 0.294 \\
\hline $\begin{array}{l}\text { Tuberculosis contact } \\
(\mathrm{n}=720 / 162 / 558)\end{array}$ & $29(4.0)$ & $7(4.3)$ & $22(3.9)$ & 0.829 \\
\hline Cavity ( $n=1741 / 219 / 1522)$ & 649 (37.3) & $115(52.5)$ & $534(35.1)$ & $<0.001$ \\
\hline
\end{tabular}

BMI, body mass index; PTB, pulmonary tuberculosis.

group B. No significant differences in the rates of other drug-resistant subgroups between group A and group B were identified ( $>00.05)$ (table 3).

\section{Comorbidities detected among retreated PTB}

Among 241 (12.5\%) patients with retreated PTB with comorbidity, extrapulmonary comorbidity accounted for $77.6 \%$ (187), pulmonary comorbidity $27.4 \%$ (66), both pulmonary and extrapulmonary comorbidity $5.0 \%$ (12), DM 51.5\% (124) and COPD 16.6\% (40). Among 504 patients with retreated PTB with drug resistance, $16.3 \%$ had comorbidity, $13.7 \%$ had extrapulmonary comorbidity and $3.4 \%$ had pulmonary comorbidity. The highest proportion of comorbidity was found for DM $(9.5 \%)$, followed by hypertension $(2.0 \%)$ and COPD $(1.8 \%)$. Of 82 patients with retreated $\mathrm{PTB}$ with drug resistance and baseline comorbidity, $87.8 \%$ (72) had only one kind of comorbidity, $15.9 \%$ (13) had pulmonary comorbidity alone, $79.3 \%$ (65) had extrapulmonary comorbidity alone, and $4.9 \%$ (4) had both pulmonary and extrapulmonary comorbidity (table 4 ).
Association between comorbidity status and drug resistance profiles of retreated PTB

According to univariable and multivariable analyses, overall RFP resistance (OR: 2.05, 95\% CI 1.43 to 2.94; aOR: $2.17,95 \%$ CI 1.41 to 3.36 ), overall SM resistance (OR: $1.48,95 \%$ CI 1.05 to 2.08; aOR: $1.51,95 \%$ CI 1.00 to 2.27 ) and MDR (OR: $1.91,95 \%$ CI 1.25 to 2.92; aOR: $1.96,95 \%$ CI 1.17 to 3.27 ) had a significant association with comorbidity $(\mathrm{p}<0.05)$. Comorbidity was significantly associated with overall INH (OR: $1.62,95 \%$ CI 1.16 to 2.26) and PDR (OR: $1.74,95 \%$ CI 1.05 to 2.87 ) in univariable analysis $(\mathrm{p}<0.05)$, but not in multivariable analysis $(\mathrm{p}>0.05)$ (table 5$)$.

\section{DISCUSSION}

This retrospective cohort study of patients with PTB in Shandong Province in China illustrates the risk factors for retreated PTB and the association between comorbidity status and drug resistance profiles among these patients 
Table 3 Drug-resistant profiles among patients with retreated PTB

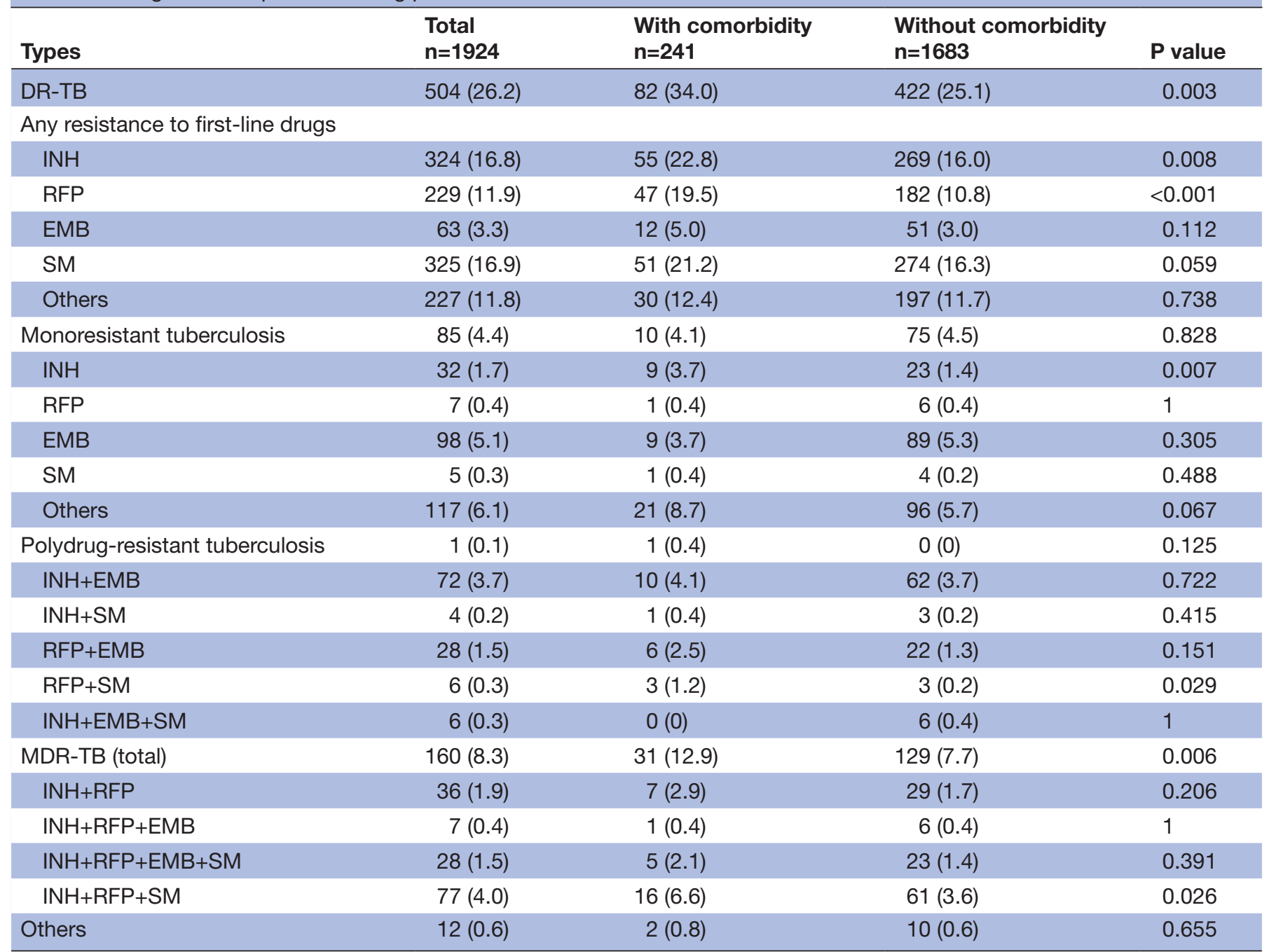

DR-TB, drug-resistant tuberculosis; EMB, ethambutol; INH, isoniazid; MDR-TB, multidrug-resistant tuberculosis; PTB, pulmonary tuberculosis; RFP, rifampicin; SM, streptomycin.

during the past 16 years. This study achieves several findings, including the following: (1) among 1924 retreated PTB cases, $26.2 \%$ were DR-TB and $12.5 \%$ had comorbidity; (2) smoking/cavity/comorbidity were risk factors for drug resistance among retreated PTB; (3) among 241 patients with retreated PTB with comorbidity, DM had the highest percentage $(51.5 \%)$, followed by COPD (16.6\%); (4) patients with retreated PTB with comorbidity were more likely to be male, to be older, with BMI $\geq 25$, to abuse cigarette/alcohol, to have clinical symptoms (expectoration, haemoptysis, weight loss) and to have DR-TB; and (5) comorbidity was also a risk factor for overall RFP resistance, overall SM resistance and MDR of retreated PTB.

Previous findings on the risk factors for DR-TB may vary in ethnicity, geographical region and study design. In this study, smoking, cavity and comorbidity were risk factors for drug resistance among patients with retreated PTB. Similarly, these factors have been reported to increase the risk of DR-TB. ${ }^{17}{ }^{20-22}$ Having TB treatment history was acknowledged as the strongest and most crucial determinant of DR-TB. While majority of the studies indicated that comorbidity (DM, HIV, COPD) and tobacco smoking were associated with DR-TB, others still found no significant relationship between them. ${ }^{14} 16{ }^{23-26}$ Based on a real-world study, TB, smoking, COPD and HIV had deleterious and synergistic relationships. ${ }^{27}$ The coexistence of TB and baseline disease may favour progression of the disease and increase the probability of drug-drug interactions or side effects. The improvement of healthassociated risk factors (eg, smoking, DM, HIV infection) was reported to mitigate TB development and mortality. ${ }^{3}$ In this study, $17.5 \%$ of cases were retreated PTB, among which $18 \%$ abuse alcohol, $20.9 \%$ were smokers, $37.3 \%$ had cavity and $12.5 \%$ had comorbidity. The high proportion of these risk factors among retreated PTB in Shandong Province deserves more attention.

DM $(51.5 \%)$ followed by COPD (16.6\%) were the most common comorbidities among patients with retreated PTB in this study. Extrapulmonary comorbidity (eg, HIV infection, DM, CKD) can facilitate the development of 
Table 4 Comorbidities detected among patients with retreated PTB

\begin{tabular}{|c|c|c|c|c|c|c|}
\hline Comorbidity & $\begin{array}{l}\text { INH } \\
n=324\end{array}$ & $\begin{array}{l}\text { RFP } \\
n=229\end{array}$ & $\begin{array}{l}\text { MDR } \\
n=160\end{array}$ & $\begin{array}{l}\text { DR } \\
n=504\end{array}$ & $\begin{array}{l}\text { Susceptible } \\
n=1420\end{array}$ & $\begin{array}{l}\text { Total } \\
n=1924\end{array}$ \\
\hline Extrapulmonary disease & 47 (14.5) & $41(17.9)$ & $28(17.5)$ & 69 (13.7) & $118(8.3)$ & $187(9.7)$ \\
\hline DM & $30(9.3)$ & $32(14.0)$ & $21(13.1)$ & $48(9.5)$ & $76(5.4)$ & $124(6.4)$ \\
\hline Hypertension & $7(2.2)$ & $2(0.9)$ & $1(0.6)$ & $10(2.0)$ & $19(1.3)$ & $29(1.5)$ \\
\hline Gastrointestinal cancer & $1(0.3)$ & $0(0)$ & $0(0)$ & $1(0.2)$ & $4(0.3)$ & $5(0.3)$ \\
\hline Hepatitis & $4(1.2)$ & $2(0.9)$ & $2(1.3)$ & $4(0.8)$ & $12(0.9)$ & $16(0.8)$ \\
\hline CRF & $0(0)$ & $0(0)$ & $0(0)$ & $0(0)$ & $1(0.1)$ & $1(0.1)$ \\
\hline CTD & $2(0.6)$ & $1(0.4)$ & $1(0.6)$ & $2(0.4)$ & $2(0.1)$ & $4(0.2)$ \\
\hline Malignancy & $2(0.6)$ & $0(0)$ & $0(0)$ & $3(0.6)$ & $16(1.1)$ & $19(1.0)$ \\
\hline Disability & $1(0.3)$ & $0(0)$ & $0(0)$ & $1(0.2)$ & $5(0.4)$ & $6(0.3)$ \\
\hline HIV infection & $1(0.3)$ & $3(1.3)$ & $1(0.6)$ & $3(0.6)$ & $0(0)$ & $3(0.2)$ \\
\hline Pulmonary disease & $11(3.4)$ & $9(3.9)$ & $5(3.1)$ & $17(3.4)$ & $49(3.5)$ & $66(3.4)$ \\
\hline Silicosis & $1(0.3)$ & $19(0.4)$ & $0(0)$ & $3(0.6)$ & $3(0.2)$ & $6(0.3)$ \\
\hline Asthma & $1(0.3)$ & $0(0)$ & $0(0)$ & $1(0.2)$ & $10(0.7)$ & $11(0.6)$ \\
\hline COPD & $6(1.9)$ & $7(3.06)$ & $4(2.5)$ & $9(1.8)$ & $31(2.2)$ & $40(2.1)$ \\
\hline Bronchiectasia & $4(1.2)$ & $1(0.4)$ & $1(0.6)$ & $4(0.8)$ & $8(0.6)$ & $12(0.6)$ \\
\hline Lung cancer & $0(0)$ & $0(0)$ & $0(0)$ & $1(0.2)$ & $4(0.3)$ & $5(0.3)$ \\
\hline Others & $1(0.3)$ & $0(0)$ & $0(0)$ & $2(0.4)$ & $2(0.1)$ & $4(0.2)$ \\
\hline Number of comorbidities & $55(17.0)$ & $47(20.5)$ & $31(19.4)$ & $82(16.3)$ & $159(11.2)$ & $241(12.5)$ \\
\hline 1 & $48(14.8)$ & $44(19.2)$ & $29(18.1)$ & $72(14.3)$ & $132(9.3)$ & $204(10.6)$ \\
\hline 2 & $6(1.9)$ & $2(0.9)$ & $2(1.3)$ & $8(1.6)$ & $21(1.5)$ & $29(1.5)$ \\
\hline$\geq 3$ & $1(0.3)$ & $1(0.4)$ & $0(0)$ & $2(0.4)$ & $6(0.4)$ & $8(0.4)$ \\
\hline Pulmonary alone & $8(2.5)$ & $6(2.6)$ & $3(1.9)$ & $13(2.6)$ & $41(2.9)$ & $54(2.8)$ \\
\hline Extrapulmonary alone & 44 (13.6) & $38(16.6)$ & $26(16.3)$ & 65 (12.9) & $110(7.8)$ & $175(9.1)$ \\
\hline Pulmonary+extrapulmonary & $3(0.9)$ & $3(1.3)$ & $2(1.3)$ & $4(0.8)$ & $8(0.6)$ & $12(0.6)$ \\
\hline
\end{tabular}

COPD, chronic obstructive pulmonary disease; CRF, chronic renal failure; CTD, connective tissue disease; DM, diabetes mellitus; DR, drugresistant; INH, isoniazid; MDR, multidrug-resistant; PTB, pulmonary tuberculosis; RFP, rifampicin.

TB by impairing the immune function and increasing bacterial loads, ${ }^{28-30}$ while pulmonary comorbidity such as COPD can promote the development of TB by damaging innate lung defence, impairing lung function and changing lung structure. ${ }^{31} 32$ As reported, DM and COPD can increase the risk of TB by 3.11 (95\% CI 2.27

Table 5 Association between comorbidity and antituberculosis drug resistance among patients with retreated PTB

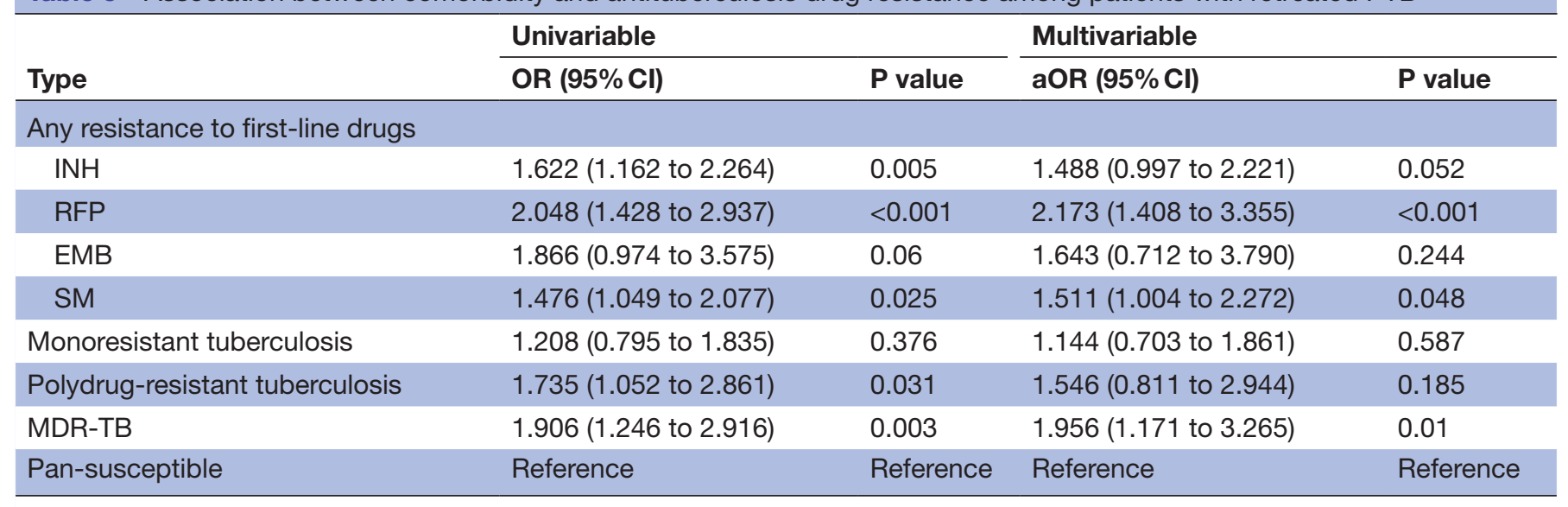

aOR, adjusted OR; EMB, ethambutol; INH, isoniazid; MDR-TB, multidrug-resistant tuberculosis; PTB, pulmonary tuberculosis; RFP, rifampicin; $\mathrm{SM}$, streptomycin. 
to 4.26 ) and 2.47 (95\% CI 2.21 to 2.76 ) compared with a control group. ${ }^{1133} \mathrm{DM}$ is one confirmed risk factor for TB which accounts for $6 \%-24 \%$ of TB burden according to geographical disparity. ${ }^{34} \mathrm{DM}$ not only increases the bacillary load of patients with active TB, but also changes the absorption and clearance of drugs. Thus it prolongs the duration of culture conversion and treatment. Similarly, TB and COPD played bidirectional roles by acting as an independent risk factor for the other. ${ }^{24}$ In this study, comorbidity not only was a risk factor for DR-TB and MDR-TB, but also contributed to overall RFP resistance and overall SM resistance among patients with retreated PTB. Previous study demonstrated that the proportions of DM among patients with TB with and without MDR were significantly different $(47.2 \%$ vs $28.1 \%, \mathrm{p}<0.05) .{ }^{16}$ Patients with TB with COPD were two times more likely to $\mathrm{die}^{23}$ and 2.5 times more likely to have MDR-TB ${ }^{6}$ than those without COPD. However, studies on the correlation between TB and coexisting diseases mainly focused on a specific type of drug resistance (MDR) and viewed all patients with TB as a whole. Studies of these correlations with other types of drug resistance among patients with retreated PTB are very limited. This study found that patients with retreated PTB with comorbidity were more likely to develop drug resistance (1.44 times), RFP resistance (2.17 times), SM resistance (1.51 times) and MDR (1.96 times) than those without comorbidity.

HIV infection was reported to be a major risk factor for TB and DR-TB in many countries. ${ }^{3}{ }^{30}$ With 95549 new patients with HIV and 15467 HIV-related deaths in 2018, China is still confronted with arduous challenges in controlling HIV. ${ }^{35}$ However, 5 of all 31 provinces accounted for approximately the whole burden of HIV in China. ${ }^{36}$ Moreover, both the incidence and HIV-related deaths in Shandong Province ranked last but one of all 31 provinces during $2004-2017 .^{35}$ In this study, only three $(0.2 \%)$ patients with retreated PTB were coinfected with HIV. Accordingly, HIV infection may not be the major factor for TB transmission in Shandong.

${ }^{24}{ }^{37}$ Previous researches showed that TB and coexisting disease shared risk factors including age, BMI and cigarette abuse. Similarly, this study showed that patients with retreated PTB with comorbidity were more likely to be male, to be older, with BMI $\geq 25$ and to abuse cigarette/alcohol than those without comorbidity. As the conditions of patients with retreated PTB, especially those with comorbidity, are complex, traditional disease-specific healthcare strategy may be less effective and multidisciplinary cooperation and integrated therapies towards high-risk populations are urgently needed.

Some information, such as DST for second-line anti-TB drugs, contact history and previous therapeutic regimen, was not available in this study, which may influence the results. Moreover, we calculated all comorbidities as a whole factor and did not specify the effect of each comorbidity in detail. In fact, previous investigations had concluded on the relationship between TB and different comorbidities inconsistently. Thus, more detailed and perspective investigations, both epidemiological and at cellular/molecular level, are urgently needed to further elaborate the contribution of comorbidity to TB/DR-TB in China.

\section{CONCLUSION}

In summary, this study finds that smoking, cavity and comorbidity are risk factors for drug resistance among retreated PTB in Shandong Province, China. Patients with retreated PTB with comorbidity are more likely to be older and with higher proportion of symptoms compared with those without comorbidity. Comorbidity is also a risk factor for overall RFP resistance, overall SM resistance and MDR among patients with retreated PTB. This study points out several directions for the control of retreated PTB: (1) the improvement of baseline health should be part of TB control; (2) bidirectional screening and coordinated treatment for both TB and comorbidity should be advocated; and (3) attention to drug resistance surveillance among patients with $\mathrm{TB}$, especially among those who had comorbidity, is imperative.

\section{Author affiliations}

${ }^{1}$ Department of Respiratory and Critical Care Medicine, Shandong Provincial Hospital Affiliated to Shandong University, Jinan, Shandong, China

${ }^{2}$ Department of Respiratory and Critical Care Medicine, Shandong Provincial Hospital Affiliated to Shandong First Medical University, Jinan, Shandong, China ${ }^{3}$ Department of Critical Care Medicine, Shandong Provincial Third Hospital, Jinan, Shandong, China

${ }^{4}$ College of Traditional Chinese Medicine, Shandong University of Traditional Chinese Medicine, Jinan, Shandong, China

Contributors $\mathrm{N}-\mathrm{nT}, \mathrm{Y}-\mathrm{fL}$ and $\mathrm{H}-\mathrm{cL}$ designed this study and drafted the initial manuscript. W-mS, J-yL, Q-yZ and T-tX collected and analysed the data. S-jL, Q-qA and S-qL coordinated and supervised data collection, and constructed the figures and tables. All listed authors have approved this manuscript.

Funding This work was supported by the Department of Science and Technology of Shandong Province (CN) (no. 2007GG30002033, 2015GSF121052 and 2017GSF218052) and Jinan Science and Technology Bureau (CN) (no. 201704100).

Competing interests None declared.

Patient and public involvement Patients and/or the public were not involved in the design, or conduct, or reporting, or dissemination plans of this research.

Patient consent for publication Not required.

Ethics approval Ethical approval for this study was obtained from the Ethics Committee of Shandong Provincial Hospital, affiliated with Shandong University, Shandong, China. Before analysis and reporting, patient records were anonymised and de-identified. The Ethics Committee waived the necessity for informed consent due to the retrospective nature of this study.

Provenance and peer review Not commissioned; externally peer reviewed.

Data availability statement № data are available.

Open access This is an open access article distributed in accordance with the Creative Commons Attribution Non Commercial (CC BY-NC 4.0) license, which permits others to distribute, remix, adapt, build upon this work noncommercially, and license their derivative works on different terms, provided the original work is properly cited, appropriate credit is given, any changes made indicated, and the use is non-commercial. See: http://creativecommons.org/ licenses/by-nc/4.0/.

ORCID iDs

Ning-ning Tao http://orcid.org/0000-0002-5506-0997

Wan-mei Song http://orcid.org/0000-0001-9690-8184 


\section{REFERENCES}

1 Marais BJ, Lönnroth K, Lawn SD, et al. Tuberculosis comorbidity with communicable and non-communicable diseases: integrating health services and control efforts. Lancet Infect Dis 2013;13:436-48.

2 Bygbjerg IC. Double burden of noncommunicable and infectious diseases in developing countries. Science 2012;337:1499-501.

3 World Health Organization. Global tuberculosis report 2019. Geneva World Health Organization; 2019.

4 Monedero I, Caminero JA. Management of multidrug-resistant tuberculosis: an update. Ther Adv Respir Dis 2010;4:117-27.

5 Perri BR, Proops D, Moonan PK, et al. Mycobacterium tuberculosis Cluster with Developing Drug Resistance, New York, New York, USA, 2003-2009. Emerg Infect Dis 2011;17:372-8.

6 Pradipta IS, Forsman LD, Bruchfeld J, et al. Risk factors of multidrugresistant tuberculosis: a global systematic review and meta-analysis. J Infect 2018;77:469-78.

7 Lönnroth K, Jaramillo E, Williams BG, et al. Drivers of tuberculosis epidemics: the role of risk factors and social determinants. Soc Sci Med 2009;68:2240-6.

8 Mulenga EM, Miller HB, Sinkala T, et al. Silicosis and tuberculosis in Zambian miners. Int J Occup Environ Health 2005;11:259-62.

9 National Collaborating Centre for Chronic Conditions (UK) CfC. Practice at NICE (UK) NIfHaCE, guidance: tuberculosis: clinical diagnosis and management of tuberculosis, and measures for its prevention and control. London; 2011.

10 Byrne AL, Marais BJ, Mitnick CD, et al. Tuberculosis and chronic respiratory disease: a systematic review. Int J Infect Dis 2015;32:138-46.

11 Jeon CY, Murray MB. Diabetes mellitus increases the risk of active tuberculosis: a systematic review of 13 observational studies. PLoS Med 2008;5:e152.

12 De La Rosa GR, Jacobson KL, Rolston KV, et al. Mycobacterium tuberculosis at a comprehensive cancer centre: active disease in patients with underlying malignancy during 1990-2000. Clin Microbiol Infect 2004;10:749-52.

13 Baker MA, Harries AD, Jeon CY, et al. The impact of diabetes on tuberculosis treatment outcomes: a systematic review. BMC Med 2011;9:81.

14 Samuels JP, Sood A, Campbell JR, et al. Comorbidities and treatment outcomes in multidrug resistant tuberculosis: a systematic review and meta-analysis. Sci Rep 2018;8:4980.

15 Crofts JP, Andrews NJ, Barker RD, et al. Risk factors for recurrent tuberculosis in England and Wales, 1998-2005. Thorax 2010;65:310-4.

16 Gómez-Gómez A, Magaña-Aquino M, López-Meza S, et al. Diabetes and other risk factors for multi-drug resistant tuberculosis in a Mexican population with pulmonary tuberculosis: case control study. Arch Med Res 2015;46:142-8.

17 Diandé S, Sangaré L, Kouanda S, et al. Risk factors for multidrugresistant tuberculosis in four centers in Burkina Faso, West Africa. Microb Drug Resist 2009;15:217-21.

18 Zhao Y, Xu S, Wang L, et al. National survey of drug-resistant tuberculosis in China. N Engl J Med 2012;366:2161-70.
19 Jing $\mathrm{H}$, Wang $\mathrm{H}$, Wang $\mathrm{Y}$, et al. Prevalence of nontuberculous mycobacteria infection, China, 2004-2009. Emerg Infect Dis 2012;18:527-8.

20 Flor de Lima B, Tavares M. Risk factors for extensively drug-resistant tuberculosis: a review. Clin Respir J 2014;8:11-23.

21 Jacobs MG, Pelissari DM, Pinto VL. Factors associated with the drug-resistant tuberculosis incidence rate in Brazil. Int J Tuberc Lung Dis 2018;22:675-80.

22 Zhao P, Li XJ, Zhang SF, et al. Social behaviour risk factors for drug resistant tuberculosis in mainland China: a meta-analysis. J Int Med Res 2012;40:436-45.

23 Inghammar M, Ekbom A, Engström G, et al. COPD and the risk of tuberculosis-a population-based cohort study. PLoS One 2010;5:e10138.

24 O'Toole RF, Shukla SD, Walters EH. Tb meets COPD: an emerging global co-morbidity in human lung disease. Tuberculosis 2015;95:659-63.

25 Wang M-G, Huang W-W, Wang Y, et al. Association between tobacco smoking and drug-resistant tuberculosis. Infect Drug Resist 2018;11:873-87.

26 Hameed S, Ahmad SR, Rahman MAU, et al. Drug resistance profile of Mycobacterium tuberculosis and predictors associated with the development of drug resistance. J Glob Antimicrob Resist 2019;18:155-9.

27 van Zyl Smit RN, Pai M, Yew WW, et al. Global lung health: the colliding epidemics of tuberculosis, tobacco smoking, HIV and COPD. Eur Respir J 2010;35:27-33.

28 Martens GW, Arikan MC, Lee J, et al. Tuberculosis susceptibility of diabetic mice. Am J Respir Cell Mol Biol 2007;37:518-24.

29 Romanowski K, Clark EG, Levin A, et al. Tuberculosis and chronic kidney disease: an emerging global syndemic. Kidney Int 2016;90:34-40.

30 Bell LCK, Noursadeghi M. Pathogenesis of HIV-1 and Mycobacterium tuberculosis co-infection. Nat Rev Microbiol 2018;16:80-90

31 Sethi S, Murphy TF. Infection in the pathogenesis and course of chronic obstructive pulmonary disease. $N$ Engl J Med 2008;359:2355-65.

32 Inghammar M, Löfdahl C-G, Winqvist N, et al. Impaired pulmonary function and the risk of tuberculosis: a population-based cohort study. Eur Respir J 2011;37:1285-7.

33 Lee C-H, Lee M-C, Shu C-C, et al. Risk factors for pulmonary tuberculosis in patients with chronic obstructive airway disease in Taiwan: a nationwide cohort study. BMC Infect Dis 2013;13:194.

34 Workneh MH, Bjune GA, Yimer SA. Prevalence and associated factors of tuberculosis and diabetes mellitus comorbidity: a systematic review. PLoS One 2017;12:e0175925.

$35 \mathrm{Xu} \mathrm{B}$, Li J, Wang M. Epidemiological and time series analysis on the incidence and death of AIDS and HIV in China. BMC Public Health 2020;20:1906.

36 Zhang L, Chow EPF, Jing J, et al. HIV prevalence in China: integration of surveillance data and a systematic review. Lancet Infect Dis 2013;13:955-63.

37 Fletcher B, Gulanick M, Lamendola C. Risk factors for type 2 diabetes mellitus. J Cardiovasc Nurs 2002;16:17-23. 